\title{
Network Pharmacology to Explore Active Components and Pharmacological Mechanism of Action of Compound Longmaining for the Treatment of Myocardial Infarction
}

Jia Li

Shanxi University of Traditional Chinese Medicine

Wenfei Wang

Shanxi University of Traditional Chinese Medicine

Diaodiao Bu

Shanxi University of Traditional Chinese Medicine Junbo Zou

Shanxi University of Traditional Chinese Medicine Yao Wang

Shanxi University of Traditional Chinese Medicine

Yanzhuo Jia

Shanxi University of Traditional Chinese Medicine

Shangshang Yu

Shanxi University of Traditional Chinese Medicine

Jiahui Zheng

Shanxi University of Traditional Chinese Medicine

Xiaofei Zhang ( $D 2051028 @ s n t c m . e d u . c n)$

Shanxi University of Traditional Chinese Medicine https://orcid.org/0000-0002-5261-6101

Changli Wang

Shanxi University of Traditional Chinese Medicine

\section{Research}

Keywords: network pharmacology, compound Longmaining decoction, myocardial infarction, GEO chip analysis, molecular docking

Posted Date: April 13th, 2021

DOI: https://doi.org/10.21203/rs.3.rs-401777/v1 
License: (c) (i) This work is licensed under a Creative Commons Attribution 4.0 International License. Read Full License 


\section{Abstract}

Background and objective: Myocardial Infarction (MI) is a cardiovascular disease with a high morbidity and mortality rate. While $\mathrm{MI}$ is currently treated with pharmaceuticals, there is a need for new treatment options: compound Chinese medicines may have unique advantages for the treatment of MI. In this study, we used network pharmacology to explore the molecular mechanisms of action of Compound Longmaining (CLMN) decoction as a potential treatment approach.

Methods: Chip data related to Myocardial Infarction were downloaded from the GEO database and genes from GSE48060 and GSE66360 chips were obtained by R language. The targets of active components of CLMN and myocardial infarction disease were predicted by network pharmacology. After intersection analysis, the core targets were obtained and functional enrichment analysis was carried out. CLMN biological activity was verified by molecular docking prior to establishing a mouse MI model to verify efficacy using hematoxylin-eosin (HE) staining and immunohistochemistry.

Results: KEGG pathway analysis showed that TNF, IL1B, PTGS2, VCAM1, and NFKBIA which mainly act on NF-kappa B pathway contribute to the anti-inflammatory effects of CLMN. Immunohistochemical analysis showed that TNF- $a$ and TRAF-2 expression levels in MI of CLMN-treated mice were decreased, while IkBa was increased $(P<0.05)$. HE staining showed CLMN reduced inflammation in mouse cardiomyocytes and decreased fibrosis.

Conclusion: CLMN regulates the NF-kappa B signaling pathway and the expression of TNF, IL1B, PTGS2, VCAM1 and NFKBIA. Chemical analysis showed that CLMN active components such as Daidzein-4,7diglucoside, rutin and puerarin may be responsible for the therapeutic effect of the decoction.

\section{Introduction}

Cardiovascular disease is the leading cause of death among urban and rural residents in China, a trend that has increased recently [1]. In 2014, there were approximately 290 million patients with cardiovascular disease, which included 2.5 million myocardial infarction cases. In the United States, there are approximately 1.5 million cases of $\mathrm{MI}$ annually with a yearly incidence rate of approximately 600 cases per 100,000 people [2]. Although the prognosis of MI has been greatly improved, it is still the main cause of morbidity and mortality in the world [3]. Therefore, it is important to continue to develop new therapeutics based upon the use of novel strategies to interrogate mechanisms involved in Ml. Following $\mathrm{MI}$, there is cardiomyocyte necrosis, the result of ischemia that causes an inflammatory response. Oxidative stress mediated by inflammatory factors damages vascular endothelial cells, and leads to plaque rupture and hemorrhage [4]. These triggering events cause serious clinical manifestations.

Traditional Chinese medicines (TCMs) have the potential advantage of low toxicity and broad spectrum activity to prevent and treat a wide variety of diseases including MI. CLMN is composed of the following natural ingredients: Pueraria lobata, Rhizoma Dioscoreae Nipponicae, Ligusticum chuanxiong, propolis. Active molecules identified in CLMN are flavonoids, puerarin, alkaloids, lactones, phenols, organic acids, 
steroidal saponins, flavonoids and terpenes [5]. It is an in-hospital preparation developed by Professor Tao Genyu of the affiliated Hospital of Shaanxi University of Chinese Medicine. Since previous studies have confirmed that CLMN decoction has a significant therapeutic effect on MI [6], we used network pharmacology combined with GEO chip analysis and molecular docking to explore which components, targets and pathways are regulated by CLMN.

Network pharmacology can provide insight into "component-target-pathways" of drugs, and can help predict the correlation between drugs and diseases [7]. Microarray analysis has been widely used to identify differentially expressed genes (DEGs) and functional pathways involved in disease progression. Lastly, molecular docking is a technique to visualize drug interactions with target proteins [8].

In this study, we used network pharmacology, microarray analysis and molecular docking to study and identify the pharmacological actions of CLMN for MI. In addition, we used docking analysis, with docking scores to characterize molecular interactions of compounds with target proteins. Finally, HE staining and immunohistochemistry were used to study efficacy and mechanisms of action of CLMN in a mouse model of MI. The flow chart of the study is shown in Fig. 1.

\section{Materials And Methods}

\section{Screening of chemical constituents and acquisition of targets of CLMN}

Traditional Chinese Medicine Systems Pharmacology (TCMSP『http://lsp.nwu.edu.cn/tcmsp.php) database [9] and scientific literature allowed us to identify CLMN chemical constituents and targets of four TCMs: Pueraria lobata, Rhizoma Dioscoreae Nipponicae, Ligusticum chuanxiong, propolis. Oral Bioavailability $(O B) \geq 30 \%$ and Drug-likeness $(D L) \geq 0.18$ [10] parameters were used for screening. The 2D structure of the active components were downloaded from PubChem

(https://pubchem.ncbi.nlm.nih.gov/) database [11] and the targets were predicted using the Swiss Target Prediction (https://www.swisstargetprediction.ch/) database [12]. The target obtained from the TCMSP database was imported into the Uniprot (https://www.uniprot.org/) database [13], and standardizes with its official name.

\section{Acquisition of disease targets}

In order to obtain gene data related to Ml, we search GeneCards (https://www.genecards.org/) [14] using the keyword "Myocardial Infarction". Microarray technology and bioinformatics analysis allowed us to identify differentially expressed genes (DEGs). In this study, we used two chips, GSE48060 and GSE66360 from the GEO (https://www.ncbi.nlm.nih.gov/geo/) database [15] and analyzed them with R language "limma" installation package. The screening conditions were adj.P.Val $\leq 0.05,|\log F C| \geq 0.5$ to screen out the DEGs of diseases and draw volcano maps. Using the "pheatmap" package, the first 20 genes identified by the most significant up-regulation or down-regulation were obtained and heat maps were drawn. 


\section{Gene mapping}

The target genes obtained from the GeneCards database and the DEGs obtained from the GEO database were intersected with the target genes corresponding to the active components of CLMN, resulting in an MI disease gene dataset. The Venn diagram between "compound-target-disease" was constructed by using the "Venn diagram" package of $\mathrm{R}$ language, and the common target genes were screened by mapping.

\section{Construction and Analysis of the protein-protein interaction (PPI) Network}

The target genes of CLMN components were introduced into the STRING (https://string-db.org/) database[16], and the research species was set as "Homo sapiens". In the setting, the highest confidence level was set to 0.9 , the free target genes were hidden, and the TSV format file of the relationship diagram was derived after obtaining the target-protein interaction relationship. We imported the TSV file into Cytoscape3.7.2 [17], adjusted the color of the nodes according to the degree value, in order to more intuitively show the important CLMN targets.

\section{Construction of drug active ingredient-core target network}

The target genes of the active components of CLMN were imported into Cytoscape3.7.2, the node representing the drug active component was set to "source node", the node representing the disease target gene was set to "target node", and the network attribute was set to "interaction type" to draw the "drug active ingredient-core target" network.

\section{GO and KEGG enrichment analysis}

The core target genes of drug-disease were transformed from Gene Symbol to entrezID, by R language, and the data was processed by "BiocManager". The bar chart and bubble chart of GO and KEGG enrichment analysis were obtained under the condition of $p<0.05$.

\section{Molecular docking}

The protein structure of the key target was downloaded from the Protein Date Bank (PDB, https://www.rcsb.org/) database [18], and we download the sdf file for the ligand (active component and positive drug corresponding to the target) from the Pubchem database. The LibDock tool of Discovery Studio4.0 software was used to dock the key target protein with its corresponding active component and positive drug, and the score was analyzed.

\section{Experimental methods}

\section{Preparation of CLMN decoction}

The CLMN decoction was prepared as described previously. $18 \mathrm{~g}$ of Pueraria lobata, $12 \mathrm{~g}$ of Chuanxiong, and $6 \mathrm{~g}$ of propolis, were added 14 volumes of water, heated, boiled twice and the filtrate was 
concentrates to $0.32 \mathrm{~g} / \mathrm{mL}[19]$.

\section{Experimental animals}

Male Balbc mice weighing 22-28 g and 10-14 weeks old were purchased from

Chengdu Dashuo Experimental Animal Co., Ltd., Chengdu, China.The experimental animal license number is SYXK (chuan) 2020-030. Animals were maintained under standard laboratory temperature and humidity conditions, and had free access to food and water throughout the study. This experiment was approved by the Animal Ethics Committee of Shaanxi University of Chinese Medicine.

Sixty mice were randomly divided into 5 groups: sham operation group, MI model group, low-dose, medium-dose and high-dose CLMN groups. The mice in the sham operation group and MI model group were given normal saline, and the mice in the CLMN group were given CLMN decoction $3.26 \mathrm{~g} / \mathrm{kg} / \mathrm{d}$ (low dose), $6.52 \mathrm{~g} / \mathrm{kg} / \mathrm{d}$ (medium dose) and $13.04 \mathrm{~g} / \mathrm{kg} / \mathrm{d}$ (high dose) for 7 days, respectively.

\section{The MI mouse model}

The MI model was established as described previously. Briefly, we opened the pericardium and located the left anterior descending coronary artery (LAD), and inserted a needle, then the coronary artery of the left anterior descending branch of the left atrium was ligated with 8/0 monofilament polypropylene suture. This resulted in permanent ischemia of the artery below the ligation line. For the sham group, the needle was inserted into the left anterior descending branch of the coronary artery without ligation after thoracotomy $[20,21]$.

\section{Tissue collection \& HE staining}

Following $\mathrm{MI}$, mice were anesthetized and the fresh heart tissue was quickly taken out and frozen in liquid nitrogen for pathological examination. Myocardial tissue was then fixed in $4 \%$ paraformaldehyde and embedded in paraffin. Thereafter, the tissue was cut into 5um thick sections and stained with hematoxylin-eosin (HE), and histopathological examination was performed under a light microscope (OLUMPUS Japan) at 200 magnification.

\section{Immunohistochemical analysis}

The myocardial tissue was cut into 5 um slices, placed in xylene, and hydrated with an ethanol gradient (100\% 95\%, 80\%, 70\%, pure water). After antigen repair, slices were incubated in $\mathrm{H}_{2} \mathrm{O}_{2}$ for 15 min in the dark, and the primary antibody diluted by $5 \%$ BSA (dissolved in PBS) was added, and slices were incubated at $4{ }^{\circ} \mathrm{C}$ overnight. The following day, the second antibody was added and incubated at room temperature for 30 min, and then washed with PBS for 3 times (5 minutes each). DAB- $\mathrm{H}_{2} \mathrm{O}_{2}$ was then used until the color develops. Slices were then incubated in hematoxylin for 2 minutes, washed with water, differentiated with hydrochloric acid and ethanol for $3 \mathrm{~s}$, washed with running water for $15 \mathrm{~min}$, and dehydrated. The expression of TNF-a, TRAF2 and IkBa was evaluated by quantitative grayscale 
scanning with a medical image analysis system after taking pictures under high magnification visual field (400x).

\section{Statistical analysis}

Data was analyzed using SPSS Statistics 26 software, and the results are expressed as mean \pm SD. When the variance was homogeneous, one-way analysis of variance was used to analyze the mean between groups, and $\mathrm{P}<0.05$ was statistically significant.

\section{Results}

\section{Collection of CLMN active ingredients and targets}

In this study, 33 active compounds were collected from the TCMSP, PubChem and Swiss Target Prediction databases, targeting 2035 proteins, including 9 active compounds in Pueraria lobata targeting 506 proteins, 3 active compounds in Rhizoma Dioscoreae Nipponicae targeting 94 proteins, 12 active compounds in Ligusticum chuanxiong targeted 443 proteins, and 9 active compounds in propolis targeting 992 proteins.

\section{Screening core targets}

3652 targets were retrieved from the GeneCards database, and the data from GSE48060 and GSE66360 chips and platform were downloaded from the GEO database. 898 significant differential genes were obtained. We drew both DEGs volcano and heat maps, as shown in Fig. 2a: GSE chip DEGs volcano map and Fig. $2 \mathrm{~b}$ : a heat map. In the volcano map, the down-regulated genes in the normal group were represented by green dots, while those up-regulated in the experimental group were represented by red dots.

There were 550 compositional targets from the TCMSP and Swiss Target Prediction databases, 2495 disease targets from the GeneCards database, and 898 DEGs from the GEO database that were imported into RStudio software. Using the "VennDiagram" package, 29 drug-disease interaction targets (ABCB1, ALDH2, RORC, PLAU, TNF, KIT, PTGS2, MMP9, CXCR1, NR3C2, NLRP3, FTO, ICAM1, VCAM1, TLR4, CCR5, MME, ADA, ADRB2, VEGFA, FOS, CDKN1A, JUN, NFKBIA, TIMP2, HMOX1, CD40LG, IL1B, C5AR1) were obtained. The results are shown in Fig. 2c.

\section{Protein interaction network (PPI) analysis}

The 29 targets described above were imported into the STRING database and were view in the network diagram "Analysis" of the database. The PPI network consisted of 29 nodes and 34 edges. The average node degree was 2.34 and the average clustering coefficient was $0.521<2.83 \mathrm{e} \sim 14$, which indicated that the target genes had potential interactions. The TSV file data of protein interaction were obtained, and then the data was imported into the Cytoscape software to construct the protein interaction network diagram, as shown in Fig. 3a. The "Network Analyzer" tool was used to score the proteins. In the Figure, 
the top 10 proteins are represented by a red oval, and the rest are represented by a blue oval. TNF, JUN, IL1B, VEGFA, CCR5, MMP9, NFKBIA, proteins with high scores play an important role in the regulation of the network and be the key targets of CLMN for the treatment of MI.

\section{Construction of "active ingredient-core target" network}

Using the "merge" tool within the Cytoscape3.7.2 software, the network diagram for 32 drug active components and $29 \mathrm{Ml}$ targets was constructed (Fig. 3b). Among them, the ovals of different colors were used to represent each active ingredient from different TCMs, and the triangle represents the disease targets. The network consists of 61 nodes and 123 edges. From the graph, it is apparent that a single active component corresponds to multiple targets, and one target can also correspond to multiple active components. Taken together, CLMN therapy for MI has characteristics of multi-components and multitargets. Using the Network Analyzer analysis tool, the top components were puerarin, Kaempferol, apigenin, daidzein and top targets were PTGS2, ABCB1, MMP9, JUN and CZCR1, suggesting that all may play an important role in the mechanism of CLMN in the treatment of $\mathrm{MI}$.

\section{GO and KEGG enrichment analysis}

Using R language, 29 common targets were selected for $\mathrm{GO}$ and $\mathrm{KEGG}$ enrichment analysis with significance of $p<0.05$. We correlated CLMN treatment with neuroinflammation, bacterial molecules, lipopolysaccharide and cells to external stimuli and regulation of the production of immunoreactive cytokines. KEGG pathway enrichment of the first 20 signal pathways indicates that CLMN treatment involves NF-kappa B, TNF, IL-17. Figure 4 represents the drawing of a GO analysis bubble chart of CLMN (Fig. 4a-c), KEGG pathway analysis bar chart of CLMN (Fig. 4d) and NF-kappa B signal pathway map enriched in the first place (Fig. 4e). Based on the above analysis, the data suggests that CLMN results in a multi-modal pharmacological profile for CLMN

\section{Molecular docking analysis}

Five important target proteins, TNF, IL1B, PTGS2, VCAM1 and NFKBIA, which are ranked first in the PPI network and enriched in the first NF-kappa B pathway, were docked with their corresponding compounds by Discovery Studio4.5 software, and compared with the positive drugs of the corresponding target proteins. The docking results are shown in Table 1, and the interaction between them is shown in Fig. 5 . The results showed that the active components Daidzein-4,7-diglucoside, rutin and puerarin had good binding affinity with target proteins TNF, IL1B, PTGS2, VCAM1 and NFKBIA.

\section{HE staining results}

The results of HE staining are shown in Fig. 6. The structure of cardiomyocytes in the sham operation group was normal and orderly, with no pathological changes. However, in the MI model group, the cardiomyocytes were injured and disordered, with a large number of inflammatory cell infiltrates, and large areas of myocardial infarction and myocardial fibrosis. With the low dose of CLMN, more inflammatory cell infiltrates were observed, but with the middle and high dose groups of CLMN, 
inflammation was alleviated, the area of myocardial infarction was significantly reduced, and cardiomyocyte fibrosis was inhibited.

\section{TNF- $a$, TRAF-2 and IkB a proteins in Myocardial Infarctions}

The results of immunohistochemical staining (Fig. 7) showed that the expression of TNF-a and TRAF-2 proteins were increased by $\mathrm{Ml}$, while lkBa protein decreased. The middle and high dose groups of CLMN significantly decreased the expression of TNF- $a$ and TRAF-2 proteins and increased the expression level of IkBa protein.

\section{Discussion}

MI causes an inflammatory response mediated by cytokines [22], which may decrease myocardial contractile function and lead to myocardial hypertrophy, fibrosis and remodeling. In this paper, based upon network pharmacology combined with GEO chip, 29 core targets of CLMN were screened, and the results of docking between the core targets and active components selected by molecular docking software were stable.

The MI mouse model was established to verify important targets of the NF-kappa B signal pathway. TNF, TRAF-2 and NFKBIA mere assayed by immunohistochemical methods, and the mechanisms of action of CLMN for the treatment of $\mathrm{MI}$ were analyzed. CLMN can regulate multiple target and pathways through Daidzein-4,7-diglucoside, rutin, puerarin. The components mainly regulate the expression of NF-kappa B signal pathway and the expression of TNF, IL1B, PTGS2, VCAM1, NFKBIA. We showed that the TNF- $a$ and TRAF-2 were significantly decreased and the expression level of IkBa (NFKBIA) protein was significantly increased in the myocardium of mice treated with CLMN.

PPI network analysis showed that TNF, JUN, IL1B, PTGS2, MMP9, NFKBIA genes have a large degree of freedom. Target proteins appear to be related and combine to play a synergistic effect of CLMN. Blocking IL1 $\beta$ signal transduction can reduce cardiac fibrosis and MMP expression by reducing leukocyte recruitment and inflammation [23]. MMP9 accelerates the removal of necrotizing cardiomyocytes by degrading collagen, fibronectin and other extracellular matrix components, but MMP9 can also prolong inflammation by preventing neutrophil apoptosis. In turn, collagen and extracellular matrix components are further degraded, which may damage tissue integrity and lead to infarction expansion, which is not conducive to left ventricular remodeling [24]. PTGS2 has been shown to have anti-inflammatory properties and regulate vascular reactivity [25].

There are 32 components in CLMN that may be responsible for the therapeutic effect of CLMN, including puerarin, kaempferol and apigenin that have high scores in active ingredient-core target interactions. Studies have found that puerarin can protect cardiomyocytes by reducing the inflammatory response in cardiomyocytes, and puerarin can promote the release of vasoactive substance NO in cardiomyocytes, increase blood flow in cardiac vessels, and attenuate myocardial injury caused by continuous ischemia [26]. Kaempferol is a flavonoid, which not only has anti-atherosclerosis, anti-inflammatory, anti-oxidative 
and antibacterial effects, but can also reduce hypertrophy, interstitial fibrosis and cardiomyocyte apoptosis [27-29]. Ren et al. [30] previously showed that apigenin can significantly inhibit the expression of miR-33 to promote the expression of ABCA1 and increase cholesterol efflux, reduce blood lipids and alleviate inflammation.

The biological process enrichment analysis of CLMN shows that target-related biological processes are mainly involved in the regulation of DNA binding transcription factor activity and response to lipopolysaccharide, bacterial molecules, neuroinflammation and cell response to external stimuli. The enrichment pathway of KEGG suggests important involvement of NF-kappa B, TNF, and IL-17, which supports the observation that the pathogenesis of $\mathrm{MI}$ is related to inflammation. TNF- $\mathrm{a}$ is considered to be one of the most important inflammatory cytokines [31]. Mature cardiomyocytes have the ability to produce TNF- $a$ and its proteins under some stress-related conditions, and TNF- $a$ can directly affect cardiac function and participate in a variety of cardiovascular pathological processes [32]. NF-kappa B pathway is the most deeply studied signal transduction pathway in the development of inflammation. NFkappa B signal pathway is triggered by activated TNF- $\mathrm{a}$. TNF- $\mathrm{a}$ activates NF-KB by binding to the TNF-R1 receptor and the TNF receptor to the dead zone, TRAF2 and serine /threonine kinase RIP. In the activated NF-kappa B signal pathway, after IkBa is phosphorylated, the nuclear localization signal on NF-KB is exposed, and NF-kB enters the nucleus from the cytoplasm and binds to the KB site on the regulatory gene promoter to induce the transcription of the target gene, thus inducing a large number of inflammatory cytokines to participate in the inflammatory response [33]. IkBa is a key protein in the NFkappa B pathway, which is necessary for the activation of NF-KB mediated by TNF- $a$. It is the main regulatory inhibitory protein of NF-KB (p50-p65) in vivo. There is a KB site in the promoter of IkB a gene, so its synthesis is also regulated by NF-KB, thus forming a negative feedback regulation of NF-KB. The results of immunohistochemistry showed that CLMN decoction significantly decreased the expression of TNF- $\mathrm{a}$ and TRAF2 and increased the expression of IkBa protein. Therefore, CLMN decoction may mainly target the NF-kappa B pathway.

\section{Conclusion}

This study systematically studied the mechanism of CLMN for the treatment of MI. A total of 33 active CLMN components were collected, 550 related targets and 3393 Ml-related disease targets were obtained. Some of the main active compounds of CLMN were screened by molecular docking, and primarily enriched to NF-kappa B, TNF, and IL-17. CLMN regulates the NF-kappa B signaling pathway and the expression of TNF, IL1B, PTGS2,VCAM1 and NFKBIA. In addition, TNF, TRAF-2 and NFKBIA, were shown to be important targets in the NF-Kappa B pathway. The study demonstrates that TCMs, CLMN in particular, had diverse pharmacologically active components, which may act synergistically to provide treatment for MI. Chemical analysis showed that CLMN active components such as Daidzein-4,7diglucoside, rutin and puerarin may be responsible for the therapeutic effect of the decoction.

\section{Abbreviations}


MI

Myocardial Infarction; CLMN:Compound Longmaining; HE:hematoxylin-eosin; TCMs:Traditional Chinese medicines; DEGs:differentially expressed genes; TCMSP:Traditional Chinese Medicine Systems Pharmacology; OB:Oral Bioavailability; DL:Drug-likeness; PPI:protein-protein interaction; GO:Gene ontology; KEGG:Kyoto Encyclopedia of Genes and Genomes.

\section{Declarations}

\section{Acknowledgements}

Not applicable.

\section{contributions}

Jia Li, Wenfei Wang and Diaodiao Bu performed the data analysis, wrote the first version of the manuscript and processed the graph and the table in the manuscript. Junbo Zou finalized the manuscript. Yao Wang, Yanzuo Jia, Shangshang Yu and Jiahui Zheng collected the data. Xiaofei Zhang and Changli Wang (corresponding author) conceived and coordinated the study. All authors read and approved the final manuscript.

\section{Funding}

This work was supported by Engineering and Technology Research Center for Application and Development of Chinese Herbal Medicine in Qinling, Shaanxi Province (20082DGC-32), the National Natural Science Foundation of China (Grant no. 81373944), Discipline Innovation team Project of Shaanxi University of Chinese Medicine (2019-YL11).

\section{Availability of data and materials}

All data are available in the manuscript and they are showed in fiqures and tables.

\section{Ethics approval and consent to participate}

The experimental animal license number is SYXK (chuan) 2020-030. This experimental was approved by Chengdu Dashuo Experimental Animal Co., Ltd., Chengdu, China and the Animal Ethics Committee of Shaanxi University of Chinese Medicine.

\section{Consent for publication}

Not applicable.

\section{Competing interests}

Authors declare no conflict of interest. 


\section{References}

1. Report on cardiovascular health and diseases in China 2019: An updated summary. Chinese Circulation Journal. 2020;35(09):833-54.

2. J BE, Paul M, Alvaro A, S BM, W CC, P CA, et al. Heart Disease and Stroke Statistics-2019 Update: A Report From the American Heart Association. Circulation. 2019;139(10).

3. Okwuosa IS, Lewsey SC, Adesiyun T, Blumenthal RS, Yancy CW. Worldwide disparities in cardiovascular disease: Challenges and solutions. International Journal of Cardiology. 2016;202.

4. Hu WX, Zhang XL, Xuan L, Chen MM, Zhang H. Correlation between serum C1q tumor necrosis factor-related protein 4 and high-sensitivity C-reactive protein levels and coronary heart disease. Journal of Hainan Medical University.1-10.

5. Wang J, Zhao CB, Tang JQ, Bu DD, Zou JB, Zhang XF, et al. Simultaneous determination of 7 components in compound Longmaining by HPLC. Central South Pharmacy. 2020;18(11):1903-5.

6. Wang CL, Wang S, Shi YJ, Wang PQ. Pharmacodynamics Experiment of Fufang Longmaining Different Ratios. Journal of Liaoning University of Traditional Chinese Medicin. 2014;16(07):7-11.

7. Hopkins AL. Network pharmacology: the next paradigm in drug discovery. Nature Chemical Biology. 2008;4(2).

8. Yuan C, Wang MH, Wang F, Chen PY, Ke XG, Yu B, et al. Network pharmacology and molecular docking reveal the mechanism of Scopoletin against non-small cell lung cancer. Life sciences. 2021;270:119105.

9. Ru J, Li P, Wang J, Zhou W, Li B, Huang C, et al. TCMSP: a database of systems pharmacology for drug discovery from herbal medicines. Journal of Cheminformatics. 2014;6(1).

10. Xu X, Zhang W, Huang C, Li Y, Yu H, Wang Y, et al. A Novel Chemometric Method for the Prediction of Human Oral Bioavailability. IJMS. 2012;13(6).

11. Sunghwan K, Jie C, Tiejun C, Asta G, Jia H, Siqian H, et al. PubChem in 2021: new data content and improved web interfaces. Nucleic Acids Research. 2021;49(D1).

12. Gfeller D, Michielin O, Zoete V. Shaping the interaction landscape of bioactive molecules. Bioinformatics. 2013;29(23).

13. Consortium TU, Alex B, Maria-Jesus M, Sandra O, Michele M, Rahat A, et al. UniProt: the universal protein knowledgebase in 2021. Nucleic Acids Research. 2021;49(D1).

14. Stelzer G, Dalah I, Stein TI, Satanower Y, Rosen N, Nativ N, et al. In-silico human genomics with GeneCards. Human Genomics. 2011;5(6).

15. Ron E, Michael D, E LA. Gene Expression Omnibus: NCBI gene expression and hybridization array data repository. Nucleic acids research. 2002;30(1).

16. Szklarczyk D, Gable AL, Lyon D, Junge A, Wyder S, Huerta-Cepas J, et al. STRING v11: protein-protein association networks with increased coverage, supporting functional discovery in genome-wide experimental datasets. Nucleic Acids Research. 2019;47(D1). 
17. Kohl M, Wiese S, Warscheid B. Cytoscape: software for visualization and analysis of biological networks. Methods in molecular biology (Clifton, NJ). 2011;696:291-303.

18. K BS, Charmi B, Chunxiao B, Sebastian B, Li C, V CG, et al. RCSB Protein Data Bank: powerful new tools for exploring 3D structures of biological macromolecules for basic and applied research and education in fundamental biology, biomedicine, biotechnology, bioengineering and energy sciences. Nucleic Acids Research. 2021;49(D1).

19. Wang S, Wang CL, Shi YJ. HPLC Fingerprint of Fufang Longmaining Decoction. Chinese Archives of Traditional Chinese Medicine. 2015;33(01):89-92+9-10.

20. Gouweleeuw L, Hovens IB, Liu H, Naudé PJW, Schoemaker RG. Differences in the association between behavior and neutrophil gelatinase-associated lipocalin in male and female rats after coronary artery ligation. Physiology \& Behavior. 2016;163.

21. Wei-Lun L, Li-Wei L, Hau-Ruey C, Chun-Ting L, Shinya Y, Shin-Huei L, et al. Sleep-related changes in cardiovascular autonomic regulation in left coronary artery ligation rats: Neural mechanism facilitating arrhythmia after myocardial infarction. International journal of cardiology. 2016;225.

22. Feng GR, Xiao L, Yan XH, Heng Y, Yu LC, Lei SX, et al. The covalent NLRP3-inflammasome inhibitor Oridonin relieves myocardial infarction induced myocardial fibrosis and cardiac remodeling in mice. International Immunopharmacology. 2020(prepublish).

23. Sager HB, Heidt T, Hulsmans M, Dutta P, Courties G, Sebas M, et al. Targeting Interleukin-1 $\beta$ Reduces Leukocyte Production After Acute Myocardial Infarction. Circulation. 2015;132(20):1880-90.

24. DeLeon-Pennell KY, Tian Y, Zhang B, Cates CA, lyer RP, Cannon P, et al. CD36 Is a Matrix Metalloproteinase-9 Substrate That Stimulates Neutrophil Apoptosis and Removal During Cardiac Remodeling. Circulation: Cardiovascular Genetics. 2016;9(1).

25. Levin G, Duffin KL, Obukowicz MG, Hummert SL, Fujiwara H, Needleman P, et al. Differential metabolism of dihomo-gamma-linolenic acid and arachidonic acid by cyclo-oxygenase- 1 and cyclooxygenase-2: implications for cellular synthesis of prostaglandin E1 and prostaglandin E2. The Biochemical journal. 2002;365(Pt 2):489-96.

26. Liu T. Efficacy of puerarin on content of serum CRP, NO and CK in rats with myocardial ischemia reperfusion injury. Clinical Journal of Chinese Medicine. 2018;10(28):12-3.

27. Liu Y, Gao L, Guo S, Liu Y, Zhao X, Li R, et al. Kaempferol Alleviates Angiotensin II-Induced Cardiac Dysfunction and Interstitial Fibrosis in Mice. Cellular Physiology and Biochemistry. 2017;43(6).

28. Yu X, Fu BZ, Guo SZ, Wang W. Effects of kaempferol and formononetin on the level of reactive oxygen species in $\mathrm{H} 9 \mathrm{C} 2$ cardiomyocytes under hypoxia / reoxygenation. Liaoning Journal of Traditional Chinese Medicine. 2017;44(01):111-3+222.

29. Zhao XS, Yang HB, Zhao YC, Liu Y. Effect of kaempferol on apoptosis of rat cardiomyocyte H9c2. Journal of Zhengzhou University(Medical Sciences). 2019;54(01):106-10.

30. Ren K, Jiang T, Zhou H-F, Liang Y, Zhao G-J. Apigenin Retards Atherogenesis by Promoting ABCA1Mediated Cholesterol Efflux and Suppressing Inflammation. Cellular Physiology and Biochemistry. 2018;47(5). 
31. Zhang X, Cui L, Chen B, Xiong Q, Zhan Y, Ye J, et al. Effect of chromium supplementation on hs-CRP, TNF- $\alpha$ and IL- 6 as risk factor for cardiovascular diseases: A meta-analysis of randomized-controlled trials. Complementary therapies in clinical practice. 2021;42:101291.

32. Giroir BP, Horton JW, White DJ, McIntyre KL, Lin CQ. Inhibition of tumor necrosis factor prevents myocardial dysfunction during burn shock. The American journal of physiology. 1994;267(1 Pt 2):H118-24.

33. Monika, Sharma A, Suthar SK, Aggarwal V, Lee HB, Sharma M. Synthesis of lantadene analogs with marked in vitro inhibition of lung adenocarcinoma and TNF-a induced nuclear factor-kappa B (NFKB) activation. Bioorganic \& medicinal chemistry letters. 2014;24(16):3814-8.

\section{Tables}

Table 1

Docking scores of target proteins with their corresponding compounds and positive controls.

\begin{tabular}{|c|c|c|c|c|}
\hline $\begin{array}{l}\text { Key } \\
\text { target }\end{array}$ & Small molecule ligand & $\begin{array}{l}\text { Docking } \\
\text { score }\end{array}$ & Positive for drugs & $\begin{array}{l}\text { Docking } \\
\text { score }\end{array}$ \\
\hline TNF & $\begin{array}{l}\text { Daidzein-4,7- } \\
\text { diglucoside }\end{array}$ & 107.769 & Chloroquine & 109.37 \\
\hline IL1B & rutin & 109.301 & Talmapimod & 105.2 \\
\hline PTGS2 & puerarin & 135.137 & \multirow{6}{*}{$\begin{array}{l}\text { Dihomo-gamma-linolenic } \\
\text { acid }\end{array}$} & \multirow[t]{6}{*}{139.496} \\
\hline PTGS2 & beta-sitosterol & 131.835 & & \\
\hline PTGS2 & Mandenol & 125.133 & & \\
\hline PTGS2 & Phenethyl caffeate & 118.007 & & \\
\hline PTGS2 & Rhamnetin & 117.062 & & \\
\hline PTGS2 & Quercetin & 112.161 & & \\
\hline ICAM1 & Butylphthalide & 68.9836 & \multirow[t]{4}{*}{ Olopatadine } & \multirow[t]{4}{*}{67.4193} \\
\hline ICAM1 & Ligustilide & 65.5129 & & \\
\hline ICAM1 & apigenin & 58.8867 & & \\
\hline ICAM1 & Kaempferol & 50.3748 & & \\
\hline NFKBIA & puerarin & 98.3206 & \multirow[t]{2}{*}{ Astaxanthin } & \multirow[t]{2}{*}{101.948} \\
\hline NFKBIA & apigenin & 84.3222 & & \\
\hline
\end{tabular}

\section{Figures}




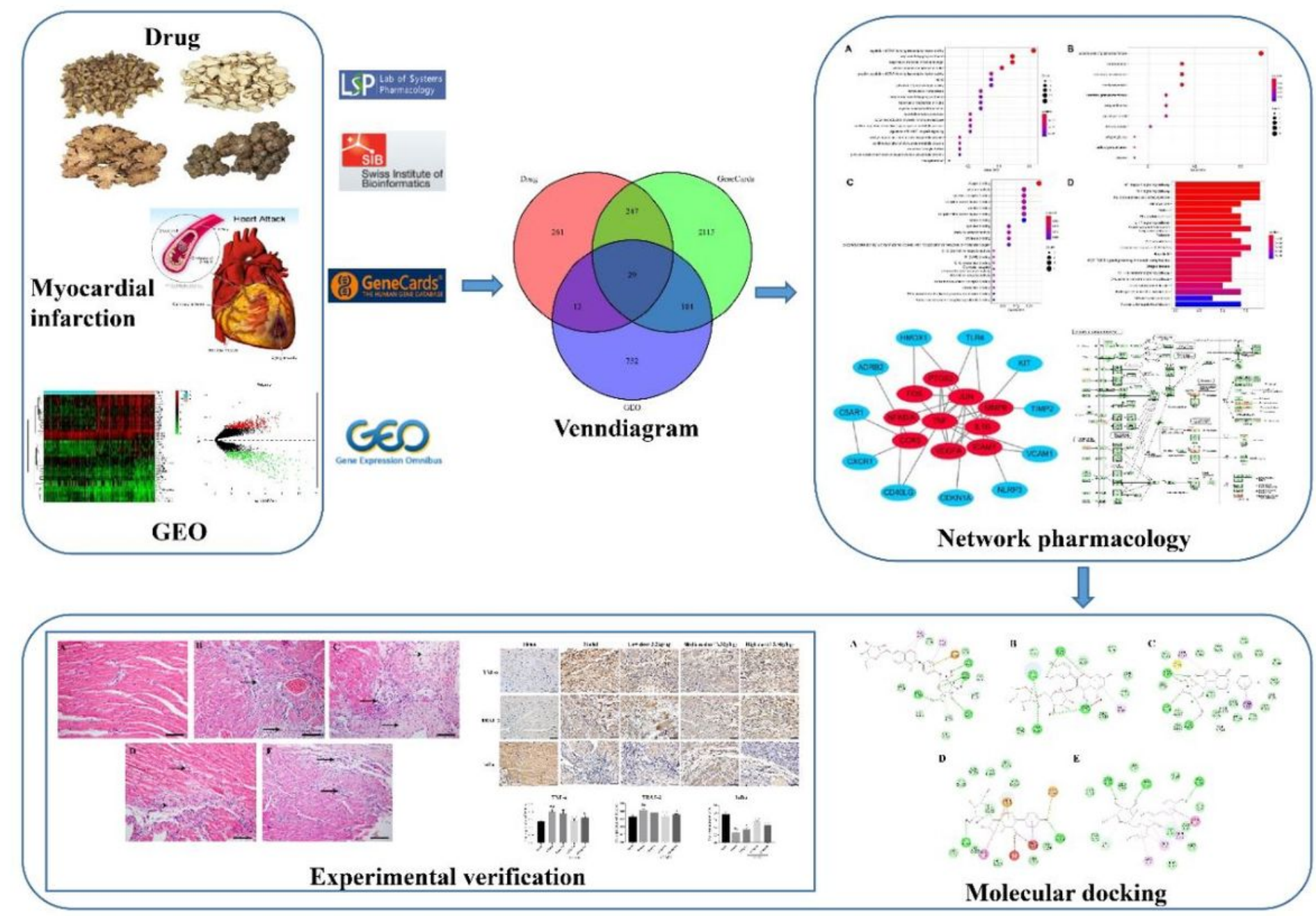

Figure 1

A detailed flow chart of the study. 


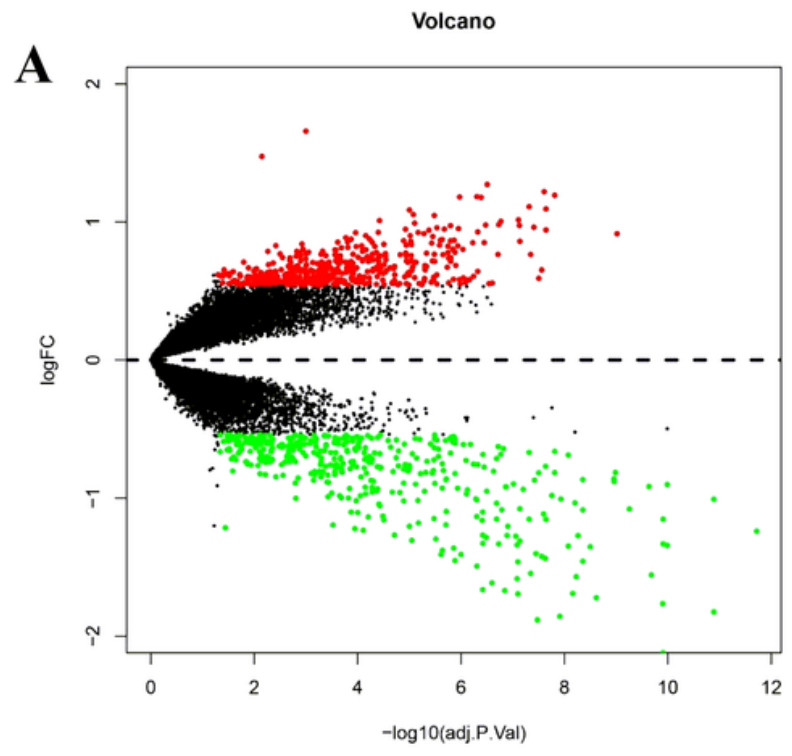

B

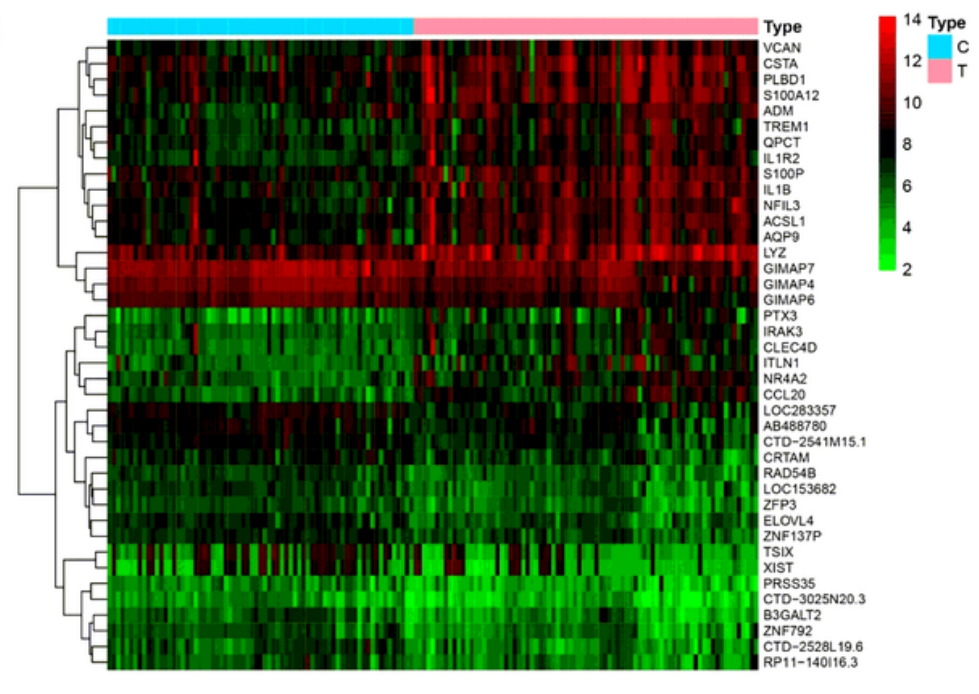

C

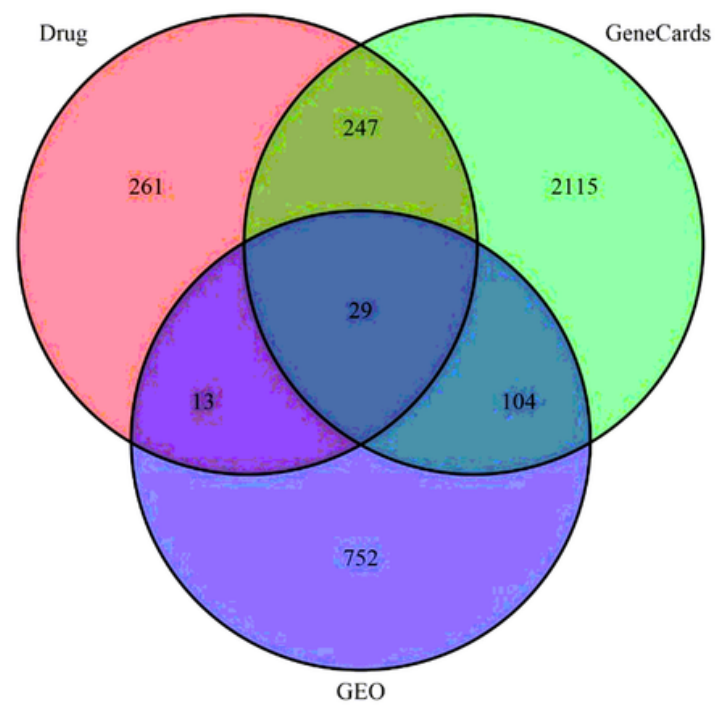

Figure 2

Screening of core targets. a GSE chip DEGs volcano map; b GSE chip DEGs heat map; c CLMN active ingredient target and disease target Venndiagram. 
A

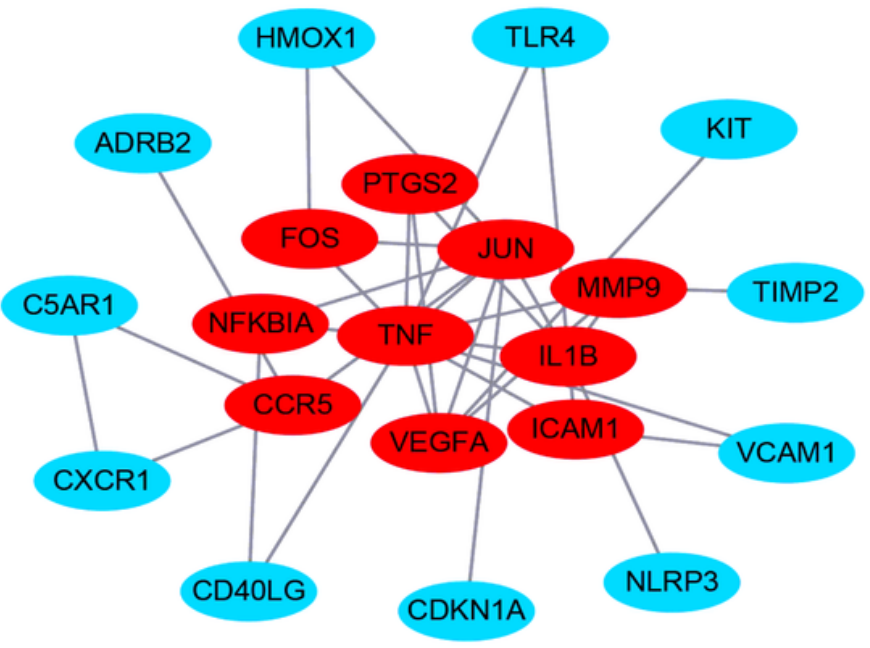

B

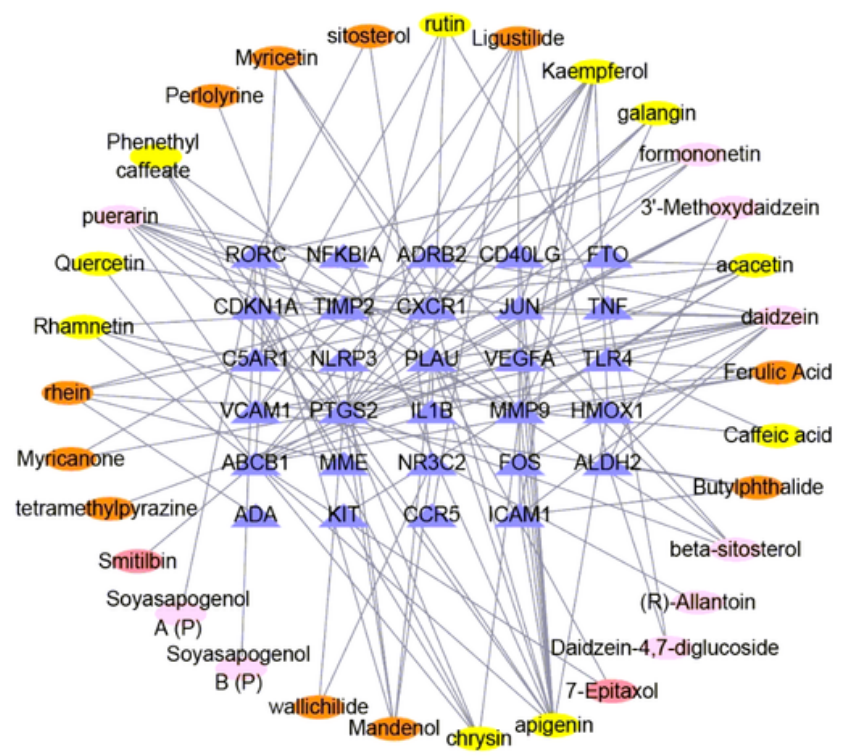

Figure 3

CLMN-MI core target network diagram. a CLMN-MI core target PPI network diagram; b CLMN active component-core target interaction network diagram.
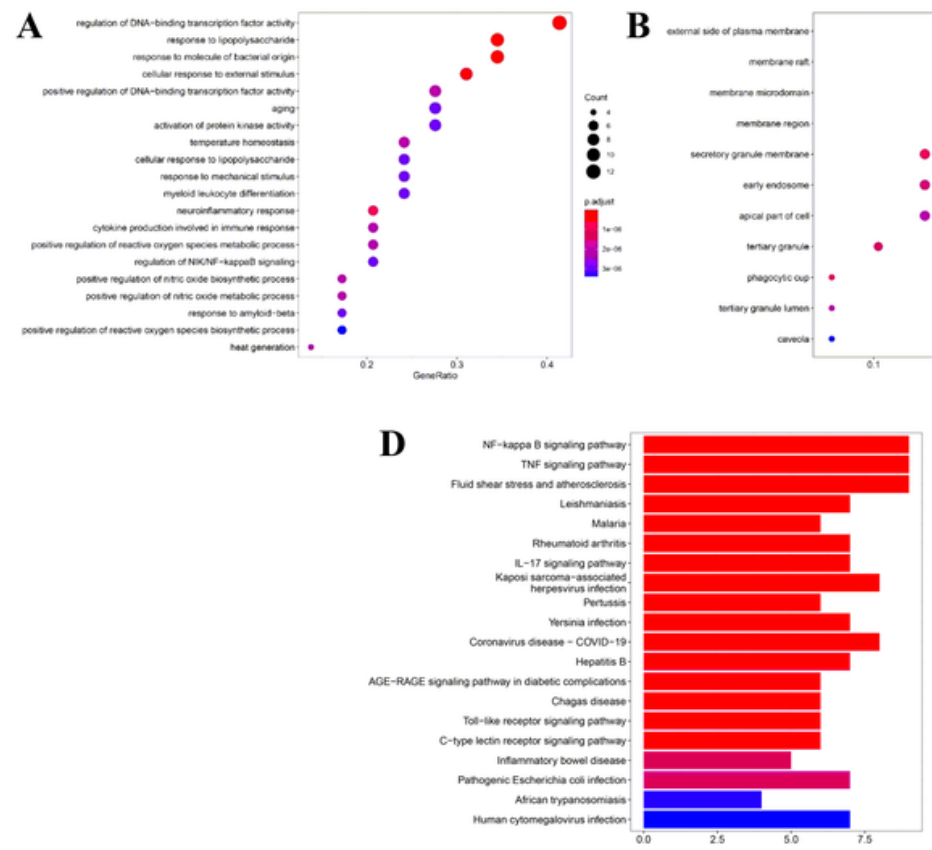
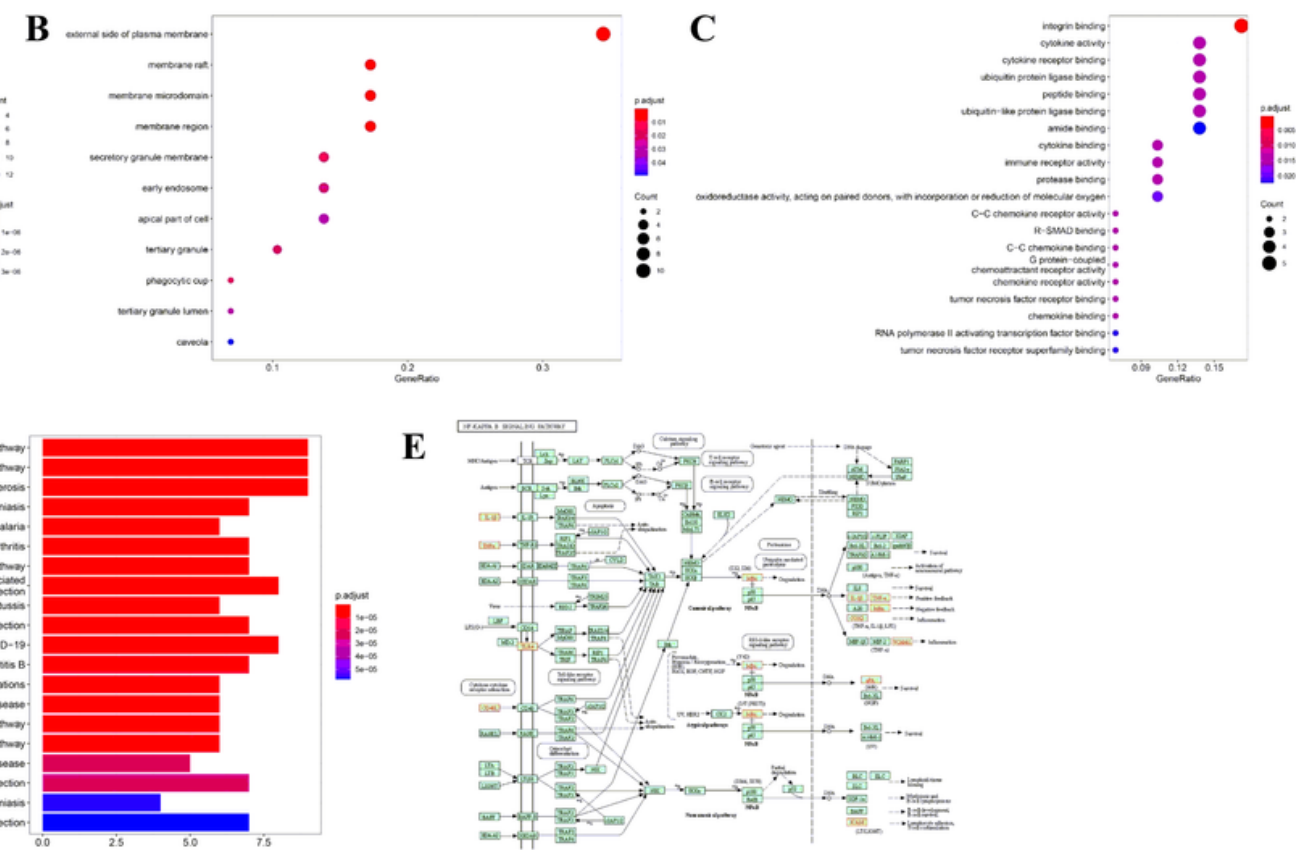

Figure 4

GO analysis and KEGG pathway diagram of CLMN in the treatment of MI. a-c GO analysis bubble chart of CLMN treatment of Ml; d KEGG pathway analysis histogram of CLMN treatment of Ml; e NF-kappa B signal pathway diagram. 

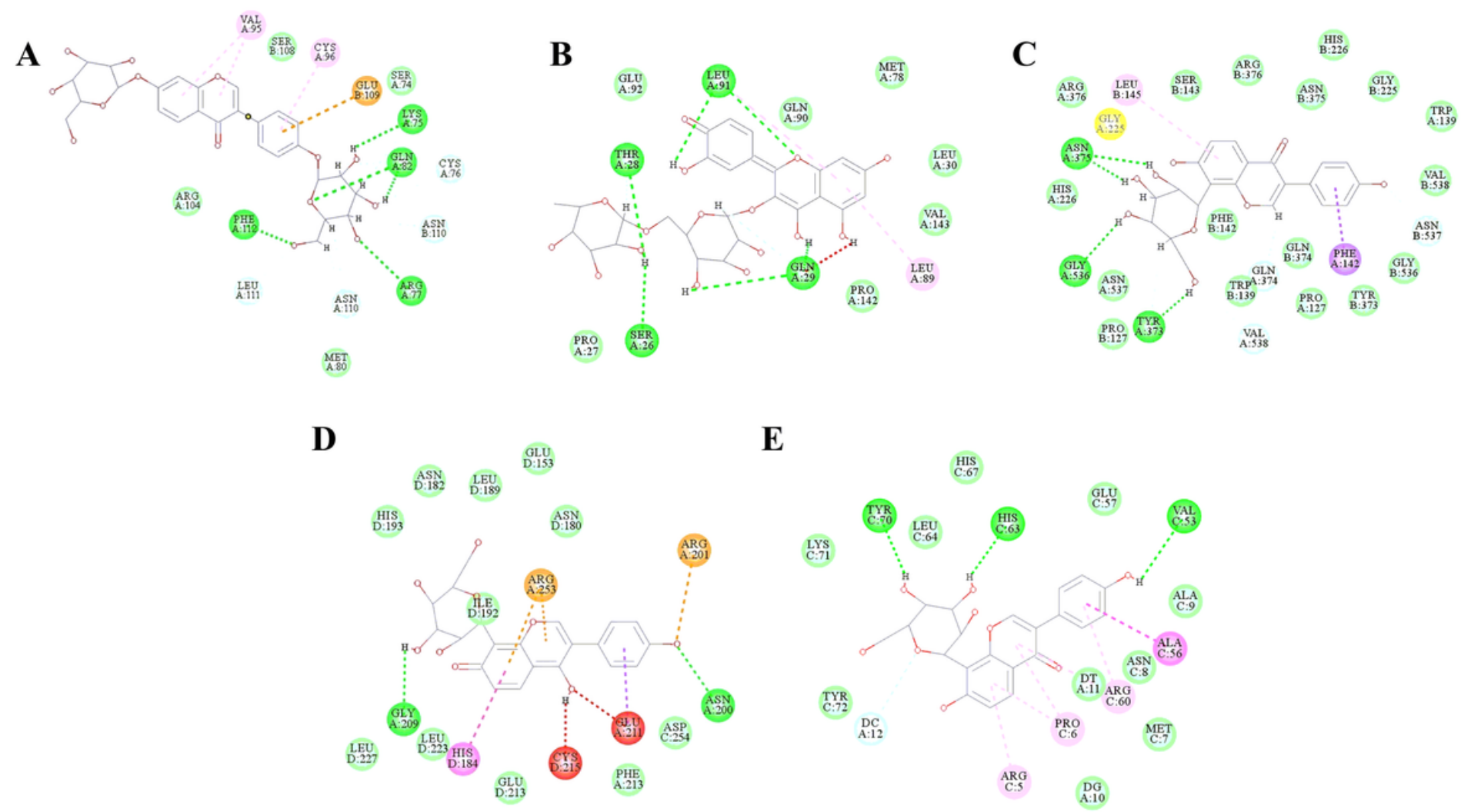

\section{Figure 5}

Docking results of key target proteins with their corresponding compounds. a TNF and Daidzein-4,7diglucoside interaction diagram; b IL1B and rutin interaction diagram; c PTGS2 and puerarin interaction diagram; d NFKBIA and puerarin interaction diagram; e VCAM1 and puerarin interaction diagram.
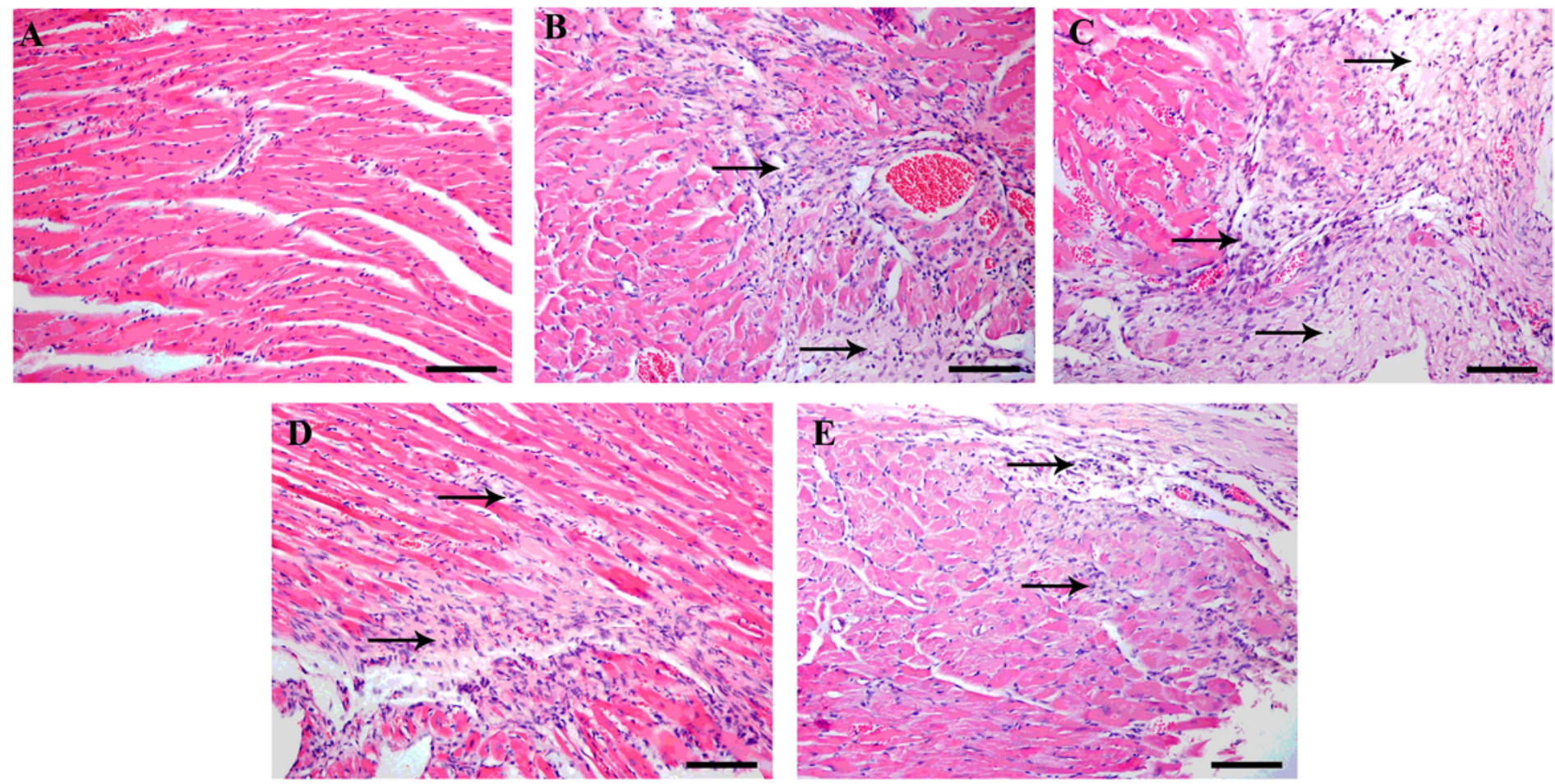


\section{Figure 6}

The effect of CLMN on the pathomorphology of myocardial tissue in rats (HE,x 200). a Sham operation group; b Model group; c CLMN low dose group; d CLMN medium dose group; e CLMN high dose group.

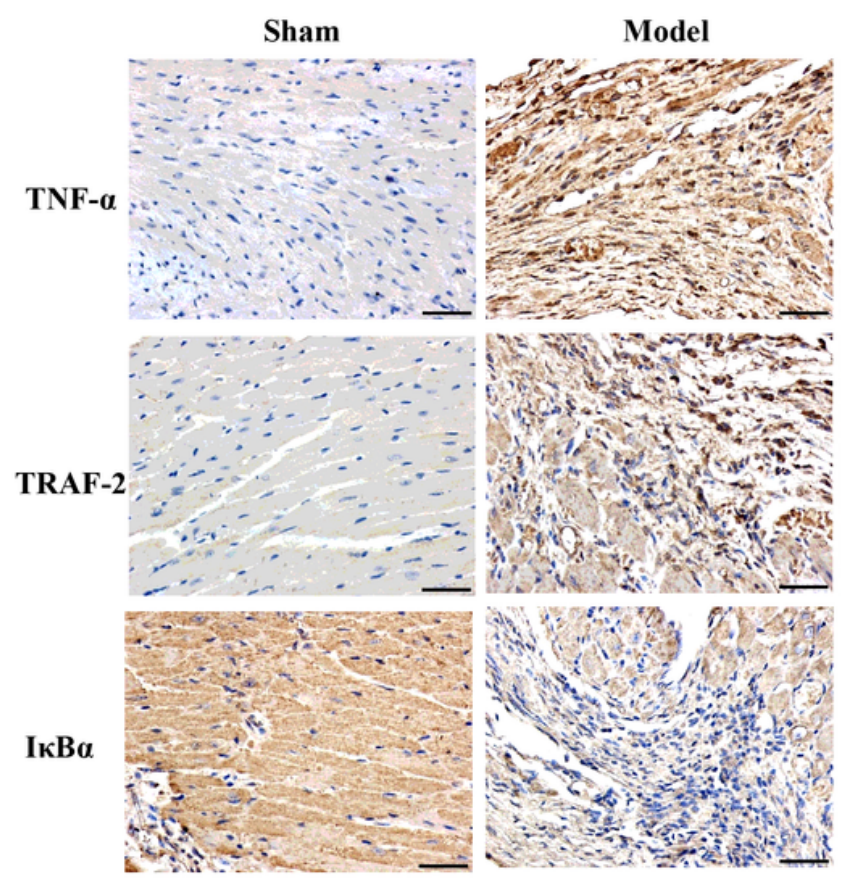

Low dose $(3.26 \mathrm{~g} / \mathrm{kg}) \quad$ Medium dose $(6.52 \mathrm{~g} / \mathrm{kg}) \quad$ High dose $(13.04 \mathrm{~g} / \mathrm{kg})$
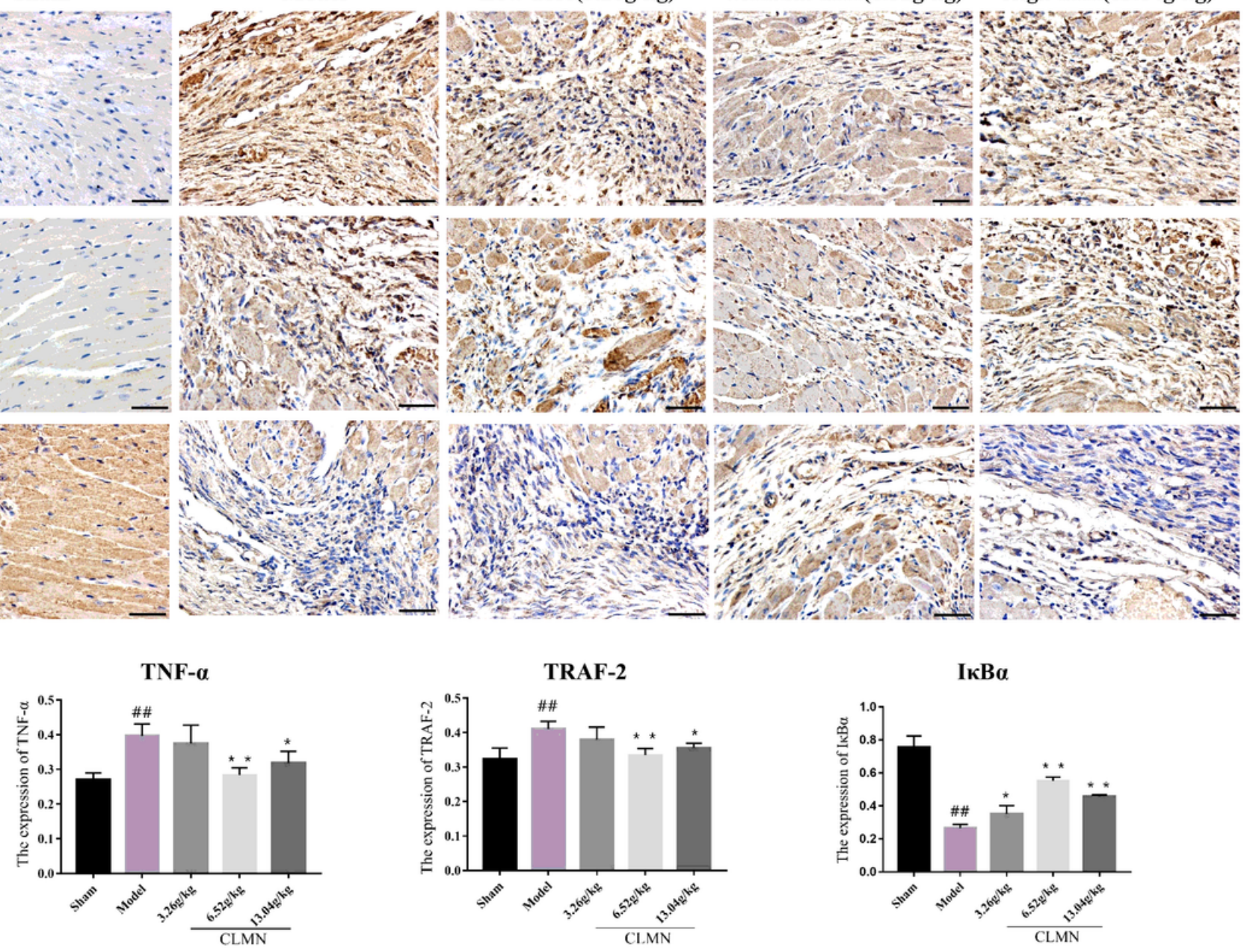

\section{Figure 7}

Effects of CLMN Pretreatment on the expression of TNF- $a$, TRAF-2, IkBa protein in Myocardial tissue of mice with $\mathrm{MI}(\mathrm{Imm}$ unohistochemistry, $\mathrm{x} 400)$ and the changes of TNF-a, TRAF-2, IkBa protein expression in Myocardial tissue of mice. 